\title{
Clonality Studies in the Analysis of Adrenal Medullary Proliferations: Application Principles and Limitations
}

\author{
Salvador J. Diaz-Cano, MD, PHD
}

\begin{abstract}
Clonality remains as the hallmark of neoplasms. A dual genetic approach using markers nonrelated (e.g., X-chromosome inactivation assays) and related to the malignant transformation (such as loss of heterozygosity analyses of tumor-suppressor genes) would provide useful clonality information from early and advanced tumor stages, respectively. Tumor progression and clonal selection would result in genetic instability and heterogeneous expression of those molecular markers related to the malignant pathway. Therefore, only the coexistence of multiple genetic abnormalities would support the clonal nature as an expression of convergent cell selection. Considering those facts, the currently available evidence on tumorigenesis and clonality in the adrenal medulla can be summarized as follows:
\end{abstract}

1. Multistep tumorigenesis defines the evolution of pheochromocytomas, as evidenced by the presence of several genetic alterations.

2. Both the significant association of nonrandom genetic alterations (specially $1 p$ and $22 q$ interstitial deletions) and the topographic accumulation of genetic deletions at the peripheral tumor compartment support a convergent clone selection for these neoplasms.

3. Although many genetic loci show nonrandom abnormalities, the most frequently involved locates on chromosome $1 \mathrm{p}$ regardless of genetic tumor background (sporadic or inherited predisposition).

4. Most pheochromocytomas should begin as monoclonal proliferations that do not always correlate with histopathologic features, particularly in inherited tumor syndromes.

5. Early histopathologic stages, described as adrenal medullary hyperplasias, are defined by hyperproliferative features in animal models and monoclonal patterns in the adrenal nodules from patients with MEN-2a.

Prescilcd in part at the 1998 Annual lindocrine society Companion Meering of the binted Srates and Canadian Aadersy of lathology, Bowon. MA, Jeb. $28,3998$.

Enstitur fir Parhologic, GSF-Forschungmentrum fir Unwelt and Gesunktheiv. Oberschleisshim (ficrmany).

Address correspondence (1) Dr. Salvador ]. Diaz-Cano,

Department of Modtid Anatomy. Royal Gondon Hospital,

Whitechapel, London $E 1$ IBB

UE (presenc address).

Eindocrine latholngy vol. ?. no. $4,301-366$, Winter 1998 c) Copvisghe 1998 by Humand

Fress the All rights of any

nature wharsoever reserved. $1046-3976 \% 88 \% 301 \cdots 316 / \$ 200$

Key Words: Pheochromocytoma; adrenal medullary hyperplasia; clonality; X-chromosome inactivation; loss of heterozygosity; clone selection; multistep carcinogenesis.

\section{Introduction}

Although many molecular features have been described in order to characterize neoplasms better, its definition is still descriptive. All these features try to distinguish neoplasms from nontumoral conditions and malignant from benign neoplasms as well. Malignant tumors (specially carcino mas) evolve through continuous and additive changes, both at genetic and phenotypic levels, ending in the complete malignant phenotype. The most widely accepted theory proposes a donal origin for them, in contrast to the heterogeneous composition in most normal tissues [1,2]. Therefore, monoclonal cell populations 
arisen from heterogeneous normal tissues strongly suggest a neoplastic nature. Different and variable success has been reported for several markers, but clonality still remains as the hallmark of neoplasms. Before any specific reference to clonality in pheochromocytomas and related lesions of the adrenal medulla, some general biologic considerations would help us to understand the strength and weakness of molecular techniques in the analysis of clonality. Those considerations will focus on the relationship among cellular kinetics, neoplastic transformation, and clonality. A brief review of the morphologic criteria of pheochromocytomas (PCC) and adrenal medullary hyperplasia will follow, and finally, the current information on clonality in the analysis of adrenal medullary proliferations will be covered.

\section{General Considerations on Malignant Iransformation and Clonality}

The term clonality has been broadly used, and it is related to the presence of cellular clones in tissues, al though that terminology can be applied at different organizational levels. Those clones represent the groups of generically identical copies of a single progenitor. The strict application of that definition would require the sequence of all DNA in order to exclude any polymorphism, but that approach is not viable. Therefore, different markers have been proposed to obtain such information using more or less complicated and simple techniques. In any case, the presence of groups of genetically identical cells must be related to proliferative advantages of those cells over the remaining. For that reason, a close relationship can be drawn between clonality and kinetics features. The expression of those features is variable from the conventional histologic level to the molecular one.

The current definition of neoplasm is descriptive and closely related to clonality. Neoplasms are defined as abnormal and self-maintained proliferations, resulting in the presence of new tissues. Those new rissues should come from a group of cells sharing a particular and common advantage. Therefore, a clear relationship between both concepts (neoplasm and clone) is present. Its application to everyday practice of pathology would also contribute to explaining the development of diagnostic criteria of neoplasm in many areas of surgical pathology. Just as an example, the diagnosis of malignant lymphomas, especially at the extranodal level, requires the presence of a monotonous proliferation of lymphoid cells that substitutes the normal tissue architecture and results in a mass. Those criteria represent, at the histologic level, the morphologic evidence of an expansive growth of neoplastic cells. Sometimes, those cellular expansions share specific phenotypes (cytoplasmic or surface markers, and so forth), which allow their identification. Such an approach would require quantification of its expression in order to distinguish the abnormal proliferation from its normal counterpart, e.g., light-chain restriction of immunoglobulin in lymphoid neoplasms or the flow cytometric analysis of surface lymphoid markers in hematologic malignancies.

On the other hand, the neoplastic transformation is a multistep process, resulting in a complex mixture of tissues with heterogeneous genetic background [1]. The results of every molecular test will depend on the timing between the evaluated biologic feature and the specific marker (Fig. 1). Simultaneously, the sensitivity and specificity of each molecular marker used to test any biologic feature, such as clonality, 
A
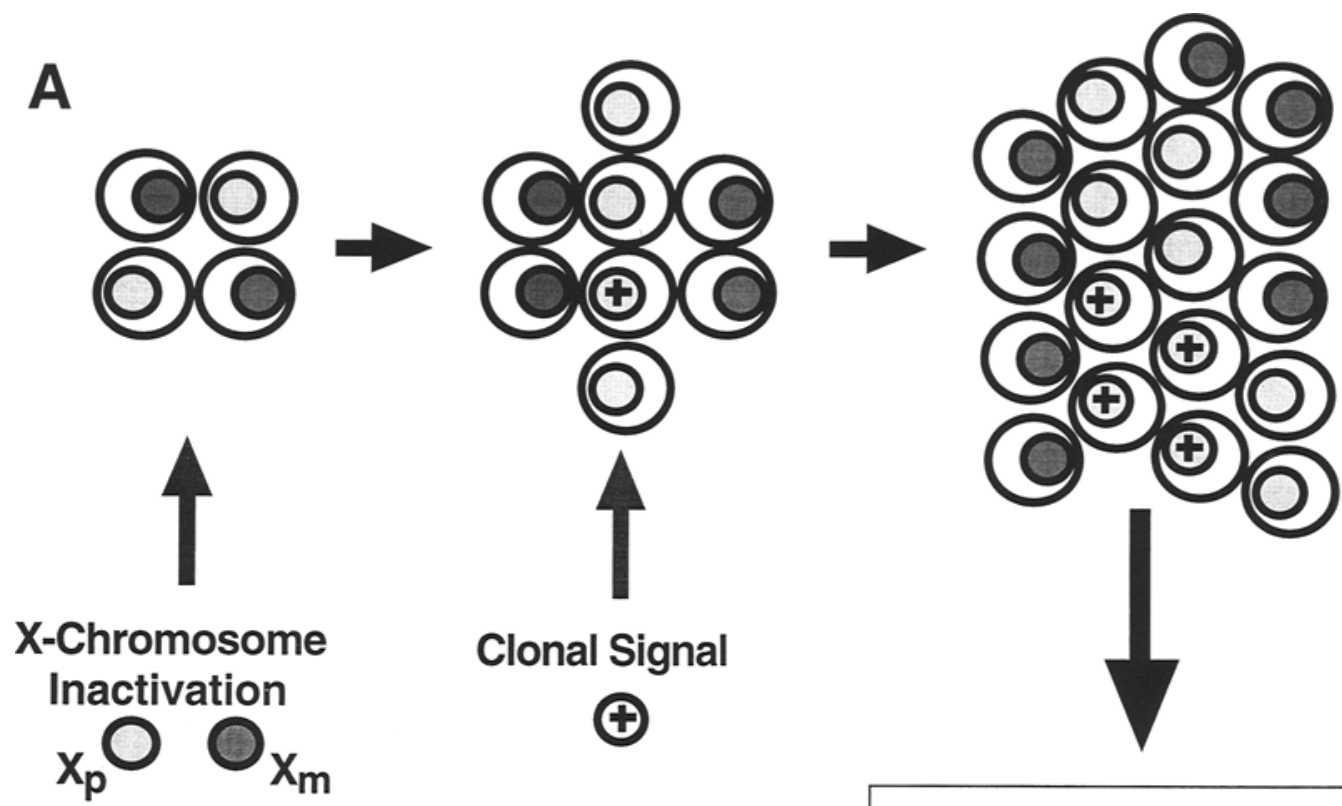

Clonal Proliferation, Monoclonal Test Pattern
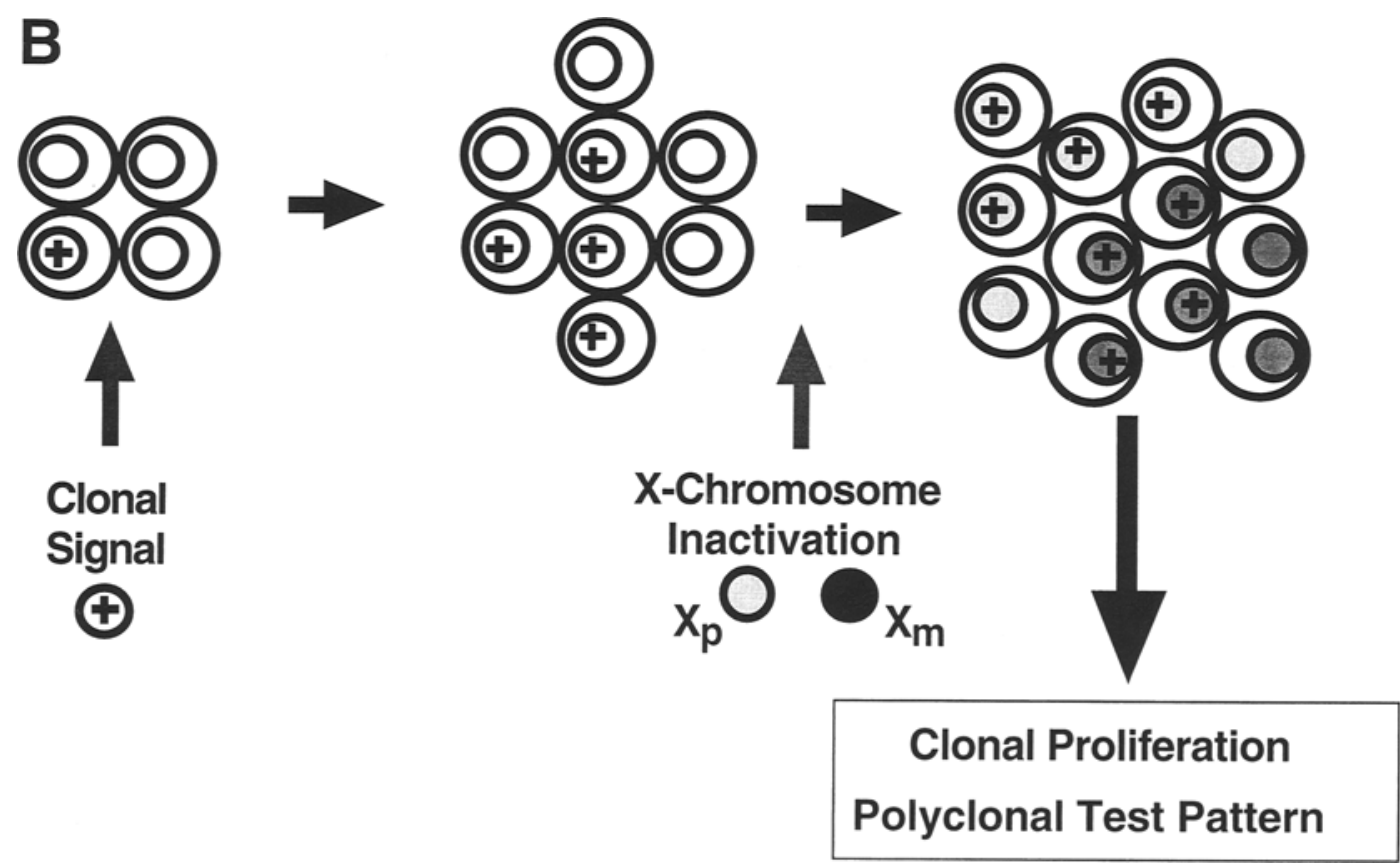

Fig. 1. Timing between the clonal signal stimulus and the $X$-chromosome inactivation $(X C 1)$. The interpretation of any test result (in this case XCI) will depend on the temporal relationship between the marker used (methylation of inactive alleles on $X$ chromosome) and the biologic feature evaluated (in this case clonality). Normally XCl takes place early before any clonal signal (labelled + in the figure), ending in monoclonal patterns (A). Those situations where the clonal signal precedes the XCl would eventually result in polyclonal patterns, even for monoclonal proliferations ("pseudopolyclonal", B). 
should be checked with samples of different and known genetic consritution. In addition, those molecular markers of clonality should be evaluated in relation to two main features, cellular kinetics and the neoplastic parhway. Any marker related to the malignant pathway will provide positive information only if that target participates in the molecular pathway of a given tumor. The current evidence suggests that malignant tumors result from the accumulation of multiple genetic events sather than from any specific sequence of generic changes [3-5]. Some mutations are more important than others, because they facilitate additional phenotypic or genotypic changes. This reason explains certain trends in the order of mutation production, although significant variation for them is the role [6]. The lack of such a sequence and the unpredictability of genetic changes in malignancies preclude the extensive clinical use of those generic markers with diagnostic and prognostic purposes. Finally, the timing between the clonal expansion and the presence and expression of malignant features is unknown. All these limitations should be considered to achieve reliable and useful information from any marker.

The final expression of any neoplasm depends on the progressive and convergent selection of cell populations, but several factors should be taken into consideration. On one hand, that selection will determine tumor progression and cellular heterogeneity. On the other hand, cellular selection is the expression of cell kinetics [7]. Considered from the genetic poinc of view, neoplasms can be characterized by multiple generic aberrations, from gross to point mutations. However, those genetic aberrations should be "fixed" on the rransformed cell before ending in a fully established malignancy. Any nonlethal genetic aberration will be "cell-fixed" if both its transmission to descendant cells is warranted and it is able to bypass the cell-repairing systems. Otherwise, any genetic alteration leading to cellular aging, differentiation, or activation of the apoptotic parhway will be nontumos-productive (Fig. 2). This process determines a complex network where a potential genetic marker will be useful when associared with kinetic advantages responsible for cellular outgrowths.

It is out of the scope of this article to provide detailed descriptions of those markers used for testing both clonality and cell kinetic [7-9]. Essentially, two types of molecular markers provide reliable results on clonality, classified according to its chromosomal location in X-linked and non- $X$-linked assays. Those markers independent of the malignant transformation pathway provide more general information, especially from the early neoplastic stages. Among them, the assays based on the random inacrivation of one X-chromosome in fermales are the most extensively used to test donality [9]. These tests target the differential expression of alleles of $X$-chromosome-linked genes (mRNA or protein analyses) or the differencial DNA methylacion on active (nonmerhylated) and inactive (methylated) alleles. Currently, the most productive marker of this sort is the human androgen receptor gene (HUMARA) owing to its high percentage of heterozygosity in the general population (around $90 \%$ ) related to the presence of a highly polymorphic trinucleotide repeat region. That polymorphic region also shows a predictable methylation pattern on sequences recognized by methylationsensitive restriction endonucleases [10], allowing allele differentiation. In fact, the information on clonality provided by both X-linked and non-X-linked assays is complementary and nonmutually exclusive, because the results from $X$-linked 


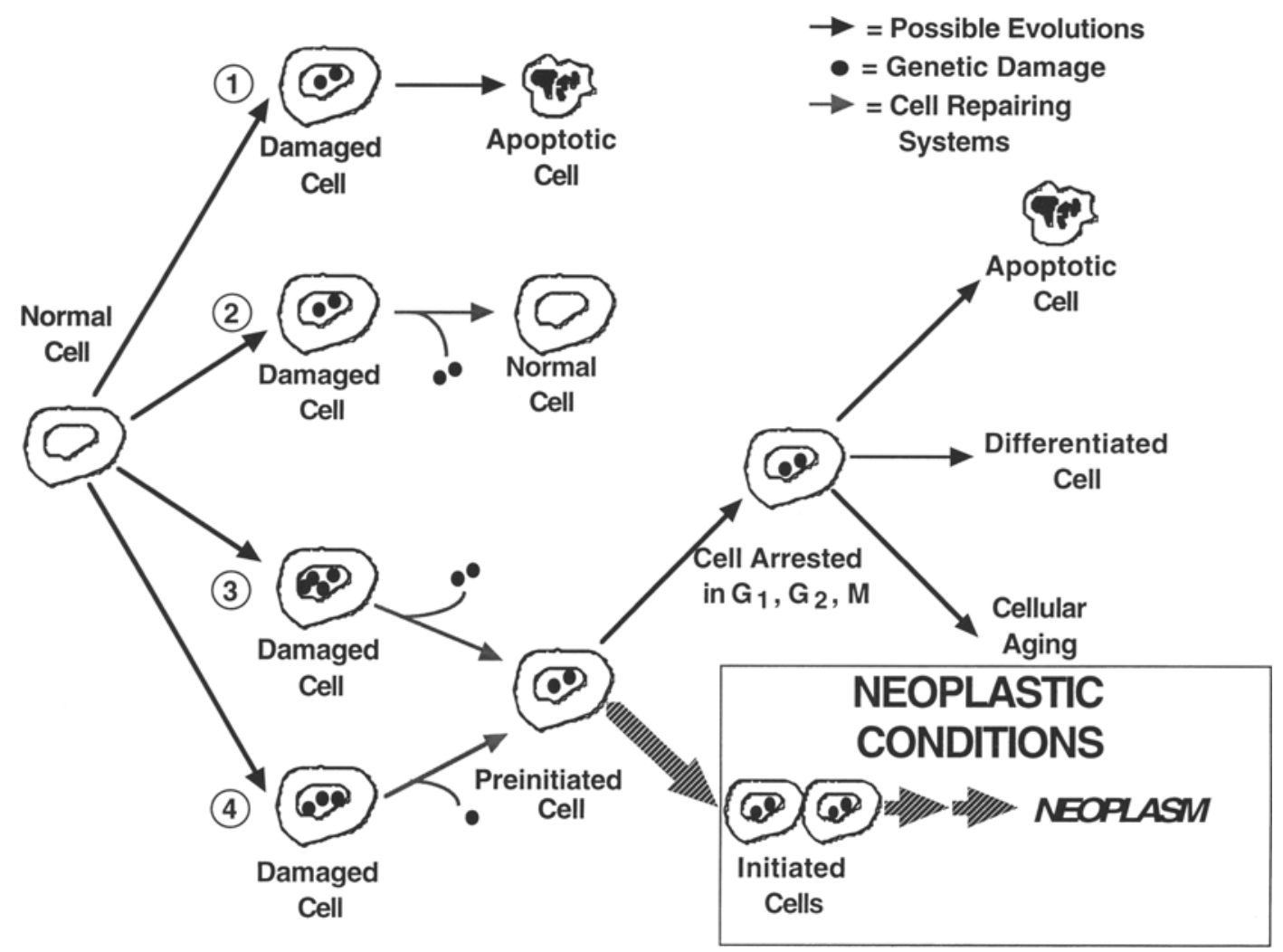

Fig. 2. DNA damage and cellular outcome. Currently, neoplasms can be considered as genetic diseases (resulted from genetic damages), which become clinically detectable after clone selection and celiular proliferation. The induction of any neoplasm requires special features for the genetic events involved in the malignant transformation. Those events (black dots) have to be resistant to the cell-repairing systems (dotted arrows), accumulated cooperatively in the cells, efficiently transmitted to descendant cells, and nonlethal (that is able to induce apoptosis). The clonal expansion of genetically damaged cells under those conditions will result in a neoplastic growth.

assays are based on genetic features usually nonrelated to the malignant transformation.

The inacrivation of one X-chromosome in females is an early event during embryogenesis, described in embryos of $16-30$ cells [11]. Two main consequences can be drawn, with both biologic and rechnical implications. First, some tissues would show the same allele inactivated and a monodonal pattern, avoiding any positive conclusion from a tumor arisen on it (noninformative case) 12,71. This pattern is variable from patient to patient and even from tissue to tissue in the same patient. For that reason, the so-called Lyonization ratio, reliable and appropriate results for any tumor or precancerous tissue samples must require embryologically related controls $[12,13]$. Second, most of somatic genetic alterations described in neoplasms will take place on cells showing one X-chromosome inactivated. Those genetic events associated with kinetic advantages and cellular overgrowths will probably determine a monoclonal methylation pattern of the $X$-chromosome if they trigger one cell or a small group of neighboring cells (Fig. 1).

Those tests based on other somatic markers potentially involved in the malignant pathway give information on the transformation mechanism. Any nonrandom genetic alteration (X-linked or not) present in most of the cumor cells will provide useful information on clonal expan- 
sion, even those as small as point mutacions. Those point mutations, regardless of their location on exons or introns (the latter, also referred to as single muclootide polymorphism, has no functional expression at mRNA and protein levels), would be related to kinetic advantages and, therefore, have been proven useful to test clonal expansions in tumors. However, only those tumors expressing that particular marker will be suitable for that analysis. Additionally, the ratio of positive results will be necessarily related with both cime-independent and time-dependent features of each tumor, e.g., histologic rype, location, grade, or stage. Those variables can be "confusing facrors" if they are not adequately evaluared. Owing to the absence of an established timing for a parricular genetic event in the transformation parbway, variably long gaps of test negativity would be demonstrated for a particular molccular marker in a given tumor (Fig. 3). That would cover the time between tumor iniciation and assay conversion, giving inconclusive results. There will not be any gap time for such conversion if the tested molecular marker represents the first mutation event in the narural history of a given neoplasm. In that case, the ress results would be identical to those provided by markers anrelated to the malignant transformation parhway, e.g., X-chromosome-inactivarion assays. In condusion, only multifactorial approaches considering different markers simultancously would appropriately provide donalivy information in tumor conditions.

\section{Pheochromocytomas and Adrenal Medullary Hyperplasias: Basic Pathology}

In general, the morphologic features of endocrine tumors barely correlate with biologic behavior. Many clinicopathologic studies make the point that cytologic features, such as nuclear pleomorphism, hypercinomatism, or increased nuclearl cyroplasmic rarios, are not necessarily related with aggressive outcome. Sometime, only the presence of metastatic tumor growths enable us to establish a malignant diagnosis [14]. Therefore, in addition, histopathological studies are required to improve both diagnosis and prognosis of these neoplasms. Orber approaches rying to detect the tumor at early steps (precancerous conditions or incipient neoplasms) go in a parallel direction to improve patient prognosis. Pheochromocytomas (PCC) fultill all these general rules.

The histopathologic differentiation of hyperplastic and ncoplastic conditions in the adrenal medulla is mainly arbitrary $[14,15]$. The first differentiaring criterion is the increase in the adrenal weight, as an expression of the increased number of chromaffin cells, with expansion of the medullary comparument into areas of the gland where it is not normally present. Morphologically, hyperplastic conditions have been classified according to their growth pattern as diffuse or nodular [16]. Addicionally, no specific and consistent criteria will distinguish big nodules within a mulrinodular background (hyperplasric condition) from small PCC (ncoplastic condirion). Some reports propose the nodule size as the main differentiating criterion and $1 \mathrm{~cm}$ as the threshold [17]. Thar size has been suggested as the criterion because it is the detection limit of most image diagnostic systems and represents the smallest size dinically derecrable. That tumor size was also selected because the smallest PCC in the first series from the Armed Forces Institute of Pathology had $1 \mathrm{-cm}$ diameter [15]. No biologic reasons are provided for that distinction. On the other hand, the knowledge available for adrenal medullary hyperplasia comes from inherited condi- 


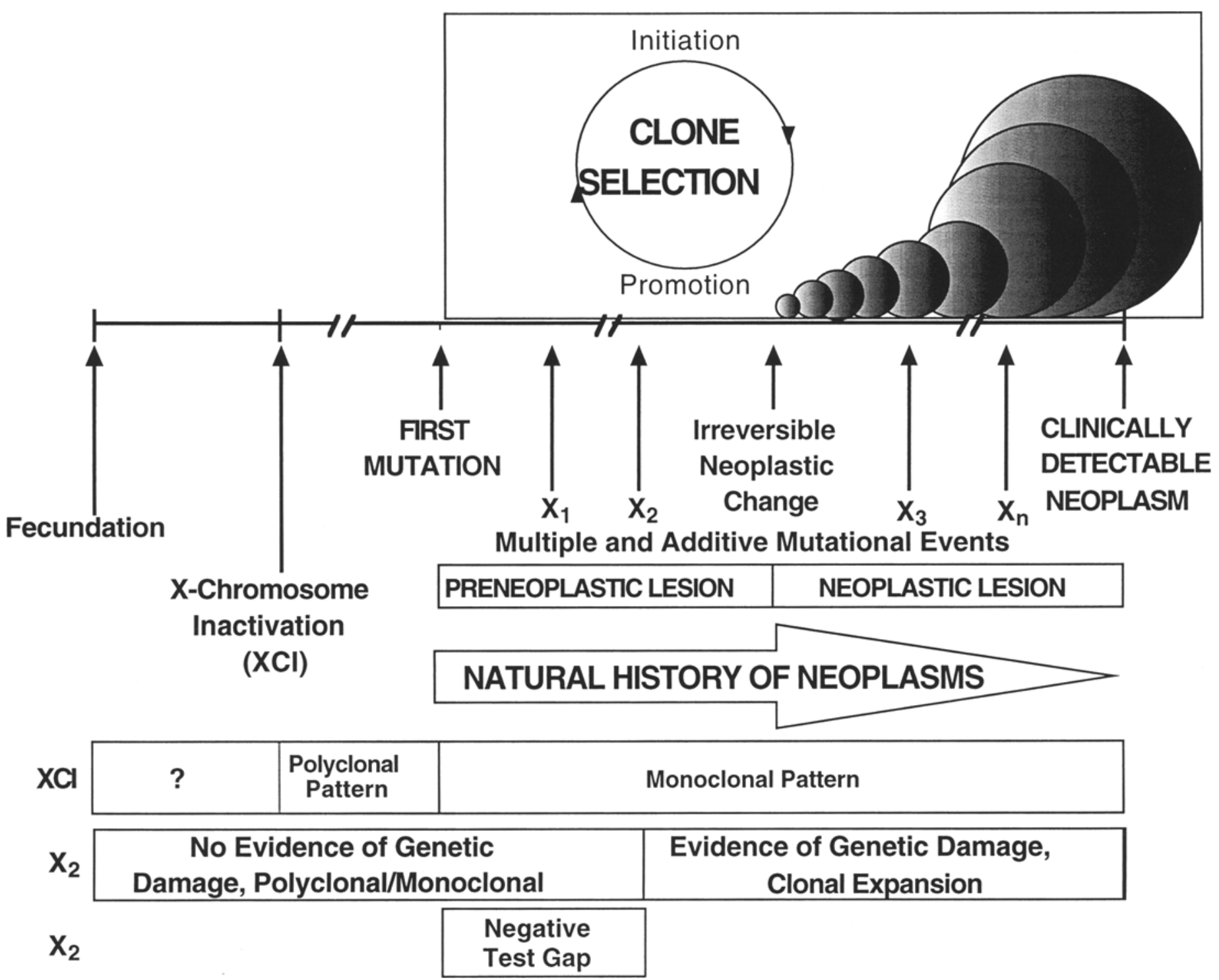

Fig. 3. Natural history of neoplasms based on clone selection and tumor progression. The natural history of neoplasms is a linear temporal expression of the convergent and endless selection process of cell clones in tumors. The clone selection results from the interaction between genetic changes (mutational events) and individual susceptibility. Progressive and cooperative changes should accumulate in cells before they are expressing a fully neoplastic phenotype. The point of irreversibility in that sequence also separates the chronologic evolution from preneoplastic into neoplastic conditions. On that background, the clonality markers will give useful information to depict the neoplastic natural history. Any particular marker will give information concerning clonality if it is involved in the malignant transformation process. Likewise, it will be concordant with XCl assays only if it represents the first mutational genetic event. All other situations will determine negative results for the marker even for already clonal proliferations (negative test gap).

rions, especially those related to MEN-2, where quancitarive analyses demonstrated utility to distinguish medullary hyperplasias from normal adrenal medulla [16]. Cytologic and architectural features have showed no significant and reliable differcnces useful for that task [15].
PCCs have been described as sporadic neoplasms or associated to other tumors as part of inherited cancer syndromes, mainly multiple endocrine neoplasia type 2 (MEN-2), neurofibromatosis, or von Hippe-Lindau disease [18]. Clinically and histopathologically, the situation is differ- 
ent for both conditions [15]. The absence of either a known generic background or a reliable screening method precludes the systematic detection of early sporadic tumors. Therefore, most of those sporadic tumors are detected in more advanced stages, although only about $10 \%$ shows malignant behavior. Additionally, no preneoplastic changes are usually associated with them. The opposite situation characterizes the inherited PCC: they are usually detected earlier owing to generic and clinical screening, and medullary hyperplasia, either diffuse or nodular, is also present.

Clinicoparhologic studies mainly define benign and malignant PCCs. Currently, a bona fide diagnosis of malignant PCC requires the demonstration of metastases, defined by the presence of tumor overgrowths in sites where chromaffin tissue is not normally present [15]. Those organs include lymph nodes, liver, and bone [14]. The presence of nonneoplastic chromaffin tissue in other organs (as a physiologic finding or as a part of hyperplastic conditions) avoids the establishment of malignant diagnoses based merely on the histopathologic evidence of such tissue. The demonstration of rruly metastatic ourgrowths would require additional studies in order to prove clonal identity in both primary and metastatic tumor [19]. Relatively clear patterns are usually shown at both ends of the pathologic spectrum (benign and malignant tumors) [20]. A slightly different situation would be expected in borderline conditions, such as locally invasive tumors $[21,22]$. 'Those tumors are characterized by variable invasion of periadrenal soft tissue, with no evidence of distant metastatic growths. Its association with lymph node metastases needs to be defined and the long-term patient outcome is unknown [15]. Other pasameters are required to obtain a full understanding on the biologic behavior of these particular tumors.

\section{Clonality Analyses of Adrenal Medullary Proliferations}

Genetic analyses of different molecular markers, especially those of polymorphic DNA regions, have provided valuable information on the parhogenesis of endocrine neoplasms, both sporadic and familial. Those polymorphic DNA areas allow reliable allele identification, and, therefore, DNA loss would be expressed as reduction to hemizygosity. According to Knudson's hypothesis, DNA loss would explain neoplastic development and progression if tumot-suppressor genes are involved $[23,24]$ A relatively common pattern for most PCCs came from those studies, showing genetic deletions (demonstrated by loss-of-heterozygosity $[\mathrm{LOH}]$ analyses) at several loci, particularly at $1 p, 3 p, 11 p$, $11 q, 17 p, 17 q$, and $22 q[25-31]$. Those interstitial DNA deletions mainly involve both known tumor suppressor genes, such as von Hippel-Lindau gene locus (3p25) [30], neurofibromatosis 1 locus (17p) [25], or p53 locus (17p) [26], and unknown tumor-suppressor genes at $\mathrm{lp}$ (at 34-36, distal to D1S73 and proximal to DIS63), $11 \mathrm{p}, 11 \mathrm{q}$, and $22 \mathrm{q}$ (distal to D22S24 and proximal to D22S1) [29-31]. Additionally, activating point mutations of genes involved in other endocrine tumors have been demonstrated in both sporadic and familial PCCs, including ret proto-oncogene [32] and the G-protein gene encoding for Gs $\alpha$ (gsp) [19].

\section{Evidences of Clonality Based} on X-Chromosome Inactivation Analysis

Preliminary results revealed monoclonal methylation pattern in the alleles of the androgene receptor gene (HUMARA assay of clonality) in $87 \%$ of informative cases from a series of sporadic PCCs [22]. After microdissection, at least two different 
samples were analyzed in each neoplasm, from the peripheral and the internal tumor areas, respectively. Both tumor areas provided concordant methylation patterns, consistent with a monodonal origin for this series of cases. The remaining $13 \%$ of informative PCCs showed balanced methylation of both HUMARA alleles, pointing to a polyclonal tumor growth. The latter group of cases corresponded to locally invasive PCCs (as defined by periadrenal soft tissue invasion) with high cell turnover rate (both MIB-1 labeling index and in situ end-labeling index received the highest scores for this series) [21]. Hiscologically, stromal overgrowh with a prominent smooth muscle differentiation (positive immunoexpression of smooth muscle type actin and desmin) was revealed by dissecting the neoplastic cell nests. Differential microdissection from several tumor locations always confirmed the initial results. Excluding the possibility of significant contamination with host normal cells by careful and repeated sampling with microdissection, those findings could be the result of (1) true polyclonal tumors, which, because of an unfinished process of neoplastic cell selection, still show coexistent cell clones descended from different progenitor, or (2) an abnormal methylation of the HUMARA locus during rumor progression (hypermethylation of the active allele) expressing pscudopolyclonal patterns in true monoclonal neoplasms.

Another method used to investigate clonality, also based on the X-chromosome inactivation, depends on the mosaicism of protein expression in the normal tissues from heterozygous women showing two isoforms of the enzyme glucose-6-phosphate dehydrogenase (G6PD) [33-35]. The original report of clonality in medullary thyroid carcinomas and PCCS associated with multiple endocrine neoplasia relies on this technique [33,34]. The authors reported the presence of only one isoenzyme in tumor tissue from patients proven to be heterozygous for that marker and concluded that the initial mutation produces multiple clones of defective cells. Thereafter, each tumor arises as a final mutation in one clone of these cells. Essentially, the proposed hypothesis claims for an initial polyclonal and nonselected proliferation of defective cells, which then turns monodonal through a process of cellular selection [36]. The histopathologic features in each stage have not been established, although theoretically adrenal medullary hyperplasia and PCC would represent the prencoplastic and neoplastic conditions, respectively.

Following the same methodology described for sporadic PCC., HUMARA clonality assay was also petformed with PCCs from members of an MEN-2a family [22]. The patients developed PCCS with no special histologic features, arisen on multinodular medullary hyperplasias. All except one cumor nodule revealed monoclonal methylation parterns, regardless of their sizes. The internodular adrenal medulla was required to show polyclonal methylation pattern in order to consider the case informative (criteria of case inclusion). Therefore, no conclusions could be obtained from the adrenal medulla with diffuse growth pattern. These results support the multistep tumorigenesis in the adrenal medulla of patients with MEN-2a $[16,17,26]$. An initial polyclonal stage would progressively evolve into monoclonal cell growths as the result of clone selection $[6,36]$. On the orher hand, they also question the validity of nodule size as diagnostic criteria to distinguish hyperplasias from neoplasms. Tumor (or nodule) size is a time-dependent patameter and revealed a low specificity for case stratification by clonal pattern. That finding can- 
not be considered surprising for neoplastic processes with early transformation events, as those reported in most inherited tumor syndromes $\{23,24\}$.

\section{Evidence of Clonality Based on Tumor Genetic Alterations and Multistep Tumorigenesis}

Although it is generally accepted the clonal evolution takes place in neoplastic transformation $[1,6]$, some clonality studies have reported controversial results in early neoplasms and preneoplastic conditions of endocrine organs |37|. Several studies essentially confirm the multistep hypothesis for PCC tumorigenesis. Although the generic mechanism of tumorigenesis has been referred to as different in pheochromocytomas and exrranadrenal paragangliomas [30], the same genetic targets seem to be involved in both sporadic and familial PCCs $[26,29]$. The general considerations mentioned above to analyze the malignant transformation and clonality [7] lead us to conclude that all these reports only provide information on clonal expansions in pheochromocytomas. The genetic homogeneity for a given marker would point to a kinetic advantage, provided by the marker itself or linked to it, that represents the basic mechanism of cell selection and tumor progression $[1,6]$. However, this isolated finding does not prove that all tumor cells come from the same progenitor and they are cruly monoclonal. The same mutagenic event can affect cells from different progenitors (Fig. 4). Therefore, the descendant cells would share the same generic abnormality (homogeneous pattern for a particular molecular marker, as expression of its clonal advantage and expansion), although they may have distinctive genetic background (different progenitors and true polyclonal origin).
Several cumor-suppressor genes have been demonstrated to play an important role in the above-mentioned clonal expansions of adrenal medullary neoplasms. As previously shown for other neoplasms, the tumor initiation and/or progression in PCCs involves multiple genes, mainly located by LOH analyses on chromosomes $1 p, 3 p, 17 p$, and $22 q[26,29-31]$. Some of these markers have also revealed significant association with clinicopathologic parameters, such as tumor volume (in the case of DNA deletions located on $1 p, 3 p$, and $17 p$ ) [26] or with a distinctive transformation pathway (i.e., 1 p34-36 and $3 p 25$ deletions have been found in 45 and $56 \%$, respectively, of PCCs, but not in extra-adrenal paragangliomas, whereas 3 p 21 deletions have been described in 50\% of extra-adrenal paragangliomas, but not in PCCs) [30]. The relative incidence of each genetic alteration is quite variable from series to series, probably in relation to the limited number of cases analyzed. However, all series agree to show $1 \mathrm{p}$ deletions as the most frequent genetic finding, although no reference is available about its timing compared with other generic abnormalities. That informarion would be especially valuable for the comparison between PCCs and adrenal medullary hyperplasias in order to determine its real nature either as an initiation-related genetic event or as a consequence of tumor progression.

On the other hand, the inherent generic instability associated with neoplasms would explain the coexistence of several genetic abnormalities $[1,6]$. In that way, a significant association between interstitial deletions on $22 q$ and $1 p$ has been reported [29]. That finding suggests that the inactivation of multiple rumor-suppressor genes is required for PCCs development and progression (multistep tumorigenesis). 


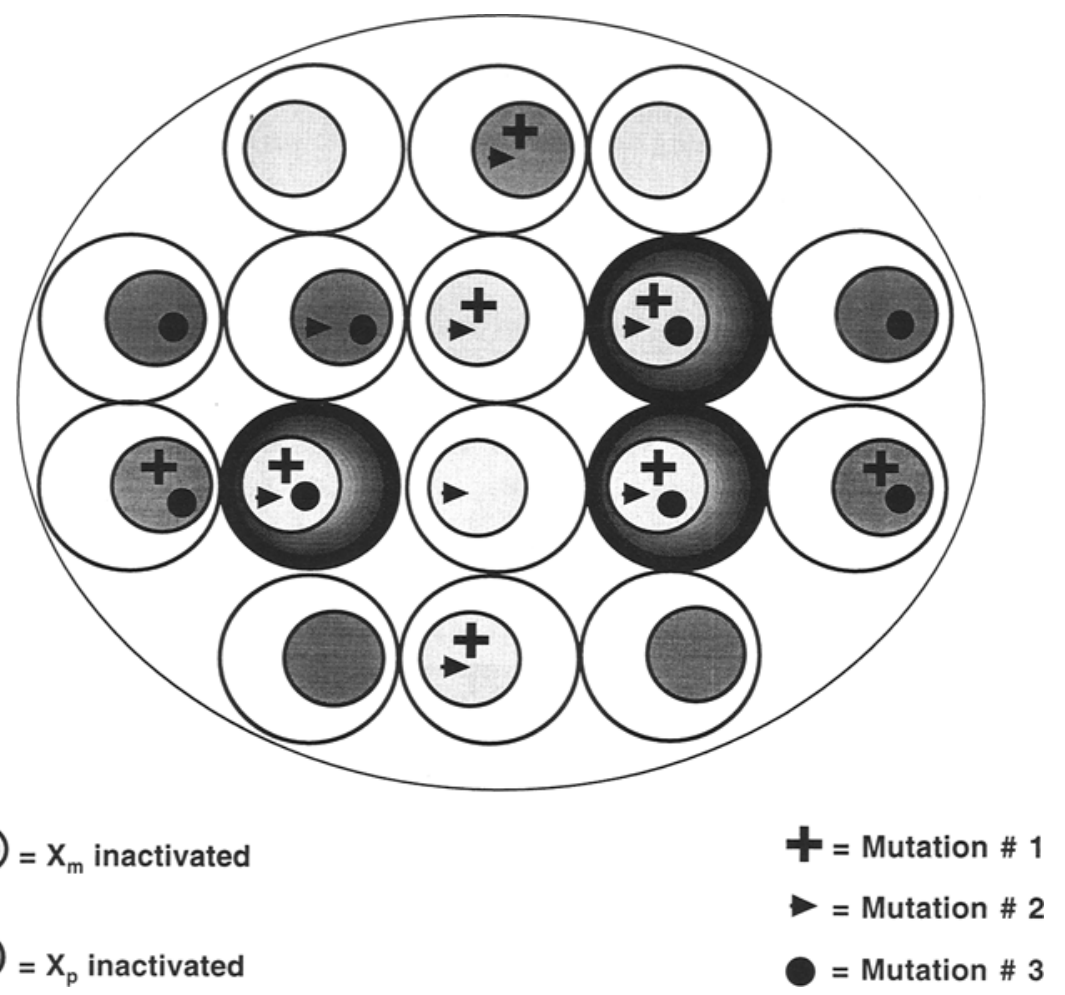

Fig. 4. Genetic alterations and clonality. LOH analyses will provide clonality information only if several markers are used. Otherwise, the presence of $\mathrm{LOH}$ in a given cell population only informs about clonal expansion.

Considering tumor heterogeneity, an additional conclusion arises from that association. From the statistical point of view, homogeneous genetic alterations would show a low probability for the random association of two or more molecular markers in tumor cell populations from different origins (polyclonal tissues). Previous reports on LOH analyses, using Southem blot hybridization [38] and PCR-based rechniques [39], have shown tandom and nontumor-related DNA deletions in $4-20 \%$ of normal tissues $[40-42]$. These percentages should be considered from two perspectives: (1) as a potential "confusing factor" in the analysis of case series, and (2) as the limiting threshold in order to interpret appropriacely the biologic significance of any associarion between DNA deletions and tumor conditions. Heterogeneity is evident among histologically similar neoplasms from different patients (intertumor heterogeneity) and among different cells of the same neoplasm at a single time (intracumor heterogeneity), as well as at different points in time (tumor progression). Considering that variability, intratumor heterogeneity for $\mathrm{LOH}$ of multiple gene loci can be exploited as biparametric markers for the analysis of clonal selection in tumor progression that LOH geneiry can be expression of either selective tumor evolucion or simple passive byproduct of other mechanisms, such as genetic instability. The association of multiple genetic alterations would become statistically less probable as the number of molecular markers increases [43]. Then the randon association of those markers would be berter explained by a convergent selective process ending in the presence of a dominant clone $[1,6]$. That selection mechanism of cell clones with relatively homogeneous genetic constitution would represent the best approach currenty available to define clonality based on tumor genetic markers. From this point of view, clonality could be considered the key element to understanding the endless biologic process of initiation and progression of tumors (cause and consequence at the same time) that also determines the otherwise linear natural hisrory of neoplasms (Fig. 3).

Some experimental resulis (personal observations) support this point of view, based on the analysis of five polymorphic DNA regions (microsatellites) located on introns of four tumor-suppressor genes (p53, Rb, WT 1, and NF1). Heterogeneous DNA loss of wild-rype alleles (LOH analyses) was revealed in informarive sporadic PCCs, involving p 53 in $45 \%, \mathrm{Rb}$ in $25 \%$, WT in $44 \%$, and NFl in $50 \%$. The comparative study of peripheral and internal tumor areas confirmed an increased accumulation of genetic deletions at the peripheral tumor compartment, expression of 
both tumor progression, and multistep tumorigenesis. At that peripheral level, two or more genes showed LOH in agreement with the generic instability of neoplasms. The low probability for random and simultaneous DNA deletion in nontransformed cell populations point to done selection, ending in outgrowths of the "selected" clone, which becomes dominant and so-called monoclonal. The final rumor picture as monoclonal proliferation thus results from a selective process on a genetically heterogeneous cell population (Fig. 4).

Other factors also contribute to neoplasiic development in endocrine organs. Somatic mutations of $G$-protein genes result in the constitutive accivation of $G$-proteins and in an overall increase of the endocrine funcrion. Along with the func tional enhancement, proliferative advantages related to the trophic effect associated with the hormonal action have also been reported in these conditions [19]. That reason would contribute to explain the frequent association berween overgrowth and hypertuncrion of endocrine organs. G-proteins represent a key element of the intracellular signal transduction linking the exracellular ligands and the final cellular response. The acrive signal transduction. normally present in most funcrional endo crine systems would explain the high sensicivity of those organs to abnomalities in that central pathway of signal transduction. That proliferative advantage would play an important sole in the kinetic evolution and progressive selection resulting in cellular transformation in those disorders. Activating mutations at codon 201 of gsp have been found in different endocrine disorders, both neoplastic (pituitary adenomas, follicular thyroid adenomas, parathyroid adenomas, chemodectomas) and nonneoplastic (parathyroid or adrenal cortical hyperplasias) [19]. The same activating mutation has also been reported in primary and metastatic PCCs as well as in extraadrenal paraganglioma from patients found to be wild-type at the germline DNA (extracted from leukocytes). That presence of concordant $G$-protein gene mutations in such a wide range of endocrine conditions and in different endoctine disorders in the same patient is consistent with a common underlying etiology.

\section{Tumor Susceptibility and Clonality in PCC of Inherited Cancer Syndromes}

The basic molecular mechanism is the same for both sporadic and inherited PCCs. However, the genetic background provides some valuable insights to understanding the general transformation process and the clonal evolution of tumors. The inherited cancer syndromes are essentially characterized by germline genetic abnormalities in certain DNA targets, frequently point mutations. Those abnormalities used to be activating point mutations targeting proto-oncogenes, such as ret in MEN-2 syndromes [32], or inactivating point mutations on cumor-suppressor genes, e.g. von Hippel-Lindau disease $[18,28]$ or neurofibromatosis 1 [25]. In both cases (activating or inactivating mutations), the basic mechanism for the clonal expansion is the proliferative advantage provided by an appropriate genetic nerwork (usually as additional somatic alterations on different genes) along with the increased susceptibility to neoplastic transformation (relared with the germline mutation). Although a similar genetic background defines a given syndrome, different transformation pathways have been described in association wich each tumor type and location. Therefore, variable relative frequencies of each generic marker have been reposted, i.e., using restriction fragment-length polymorphism analysis, Ip $\mathrm{LOH}$ has been found in all 
PCCs associated with MEN-2 syndromes (67\% including sporadic and Von HippelLindau PCCs), whereas it was detected in only $13 \%$ of MEN-2-related medullary thyroid carcinomas [26]. The germline abnormalicy provides an increased susceptibility for tumor development that modulated by the cellular environment, will only end in neoplasms if multiple genetic loci are also involved (multistep cumorigenesis). Regardless of the predisposing genetic factor, chromosome lp deletions are again the most frequently found molecular markers, as mentioned for sporadic PCCs (see Evidence of Clonality Based on Tumor Generic Alterations and Multistep Tumorigenesis) $[26,29,31]$.

The genetic background also modulates and determines the molecular mechanism involved in the transformation process. The most frequent inactivation mechanism of tumor-suppressor genes in PCCs involves both an inactivating point mutation in one allele (missense or nonsense mutations usually present as a germline defect) and the loss of the wild-type allele $[26,27]$, analogous with the model proposed for different inherited malignant tumors $[23,24,41$, $42,44,45]$. However, additional inactivating mechanisms should be considered when no DNA loss of the wild-type allele is revealed. Such mechanisms include intragenic somatic mutations in the wildrype allele (also called homozygous inactivation) and hypermethylation of the tumor-suppressor gene as described for von Hippel-Lindau gene $[28,46,47]$. On the other edge of the spectrum, several activating mutations in the ret protooncogene characterize different types of MEN-2 syndromes and familial medullary thyroid carcinomas (FMTC) [48]. Recently, a mutation of ret codon 768 in exon 13 was found to segregate with the FMTC phenotype (multiple cases of medullary thyroid carcinomas or $\mathrm{C}$-cell hyperplasia), but not with the adrenal medullary hyperplasia in a large multigenerational family [32]. Those preliminary data should provide information on additional factors responsible for the development of specific tumor types. However, these results should be taken with caution because of the short number of cases analyzed; in that particular series, only two individuals revealed isolated adrenal medullary hyperplasia.

\section{Clonality and Proliferation Features in Adrenal Medullary Hyperplasias}

As mentioned above, there is no general agreement on the morphologic criteria to distinguish neoplastic (incipient pheochromocytomas) from nonneoplastic prolifetations (hyperplasias) in the adrenal medulla [15]. On the other hand, most of our knowledge on adrenal medullary hyperplasias comes from a restrictive group of pathologic conditions, such as experimental pathology (in vitro and in vivo studies) and, to a lesser degree, from human pathology (in the case of inherited cancer syndromes). Those factors make it more difficult to obtain reliable and clinically relevant conclusions from the molecular analyses, useful for general application. Although no established conclusions are available at the molecular level, the proliferative response of the adrenal medulla is better known, at least under specific and controlled conditions [49-52].

Current evidences suggests that chromaffin cell proliferarion in adult rats is regulated by a combination of hormonal and neurogenic signals $[49,50]$. The adrenal medulla is innervated by several nerve fibers, which stimulate the secretion of catecholamines. Therefore, after chronic administration of a wide variety of catecholamine-depleting pharmacologic agents, such as the antihypertensive agent reserpine, the releasing of negative feedback 
controls increases neurogenic stimulation on chromaffin cells [50]. The reflexively increased neurogenic stimulation of chromaffin cells is intended to meet the physiological needs of catecholamine synthesis, and would result in adrenal medullary hyperplasia and neoplasia. Catecholamine depletion would be compensated through. mechanisms that normally adjusr cell number, increasing the cell turnover rate and ending in chromaffin cell outgrowths [50,52]. Many strains of rats develop similar changes spontaneously in the course of aging. These models have revealed, using different methods (mitotic figure counting and incorporation of 5-bromo-2'-deoxyuridine into replicating nuclei), a significant increase in the proliferation indices after reserpine stimulation, which can be partially prevented by adrenal denervation $[49,52]$. Denervation also causes a significant decrease in the proliferation index in nonstimulated animals. This hyperproliferative stage is postulated as a prelude to neoplastic transformation. The chronic persistence of these signals and superimposed abnormalities would lead to the selection of specific cell clones, which becoming dominant, explain the developmene and progression of neoplasms [50].

\section{Conclusions}

The currendy available evidence on tumorigenesis and clonalicy in the adrenal medulla can be summarized as follows:

1. Multistep cumorigenesis defines the evolution of pheochromocytomas, as evidenced by the presence of several genetic alterarions.

2. Both the significant association of nonrandom genetic alterations (specially $1 p$ and $22 q$ incerstitial deletions) and the ropographic accumularion of genetic delerions at the peripheral tumor com- parment support a convergent clone selection for these neoplasms.

3. Although many genetic loci show nonrandom abnormaliries, the most frequently involved genes locate on chromosome lp, regardless of generic tumor background (sporadic or inherited predisposition).

4. Most pheochromocytomas should begin as monoclonal proliferations that do not always correlate with histopathologic features, particularly in inberited tumor syndromes.

5. Early histopathologic stages, described as adrenal medullary hyperplasias, are defined by hyperproliferative features in animal models and monoclonal patterns in the adrenal nodules from patients with MEN-2a.

\section{References}

1. Nowell PC. The clonal evolution of tumor cell populations. Science 194:23-28, 1976.

2. Fialkow PJ. Clonal origin of human tumors. Biochim Biophys Acta 458:283$321,1976$.

3. Califano I, van der Riet P, Westra W, et al. Genetic progression model for head and neck cancer: Implication for field cancerization. Cancer Res 56:2488-2492, 1996.

4. Jones PA, Droller MI. Pathways of development and progression in bladder cancer: New correlations between clinical observations and molecular mechanisms. Semin Urol 11:177. $192,1993$.

5. Vogelstein B. Fearon ER. Hamilton SR, er al. Generic alterations during colorectal tumor development. $N$ Engl \} Med 319:525 $532,1988$.

6. Hellman $\$$. Oarwin's dinical relevance. Cancer 79:2275-2281, 1997.

7. Diaz-Cano SI, Wolfe HJ. PCR-based techniques for clonality analysis of neoplastic progression. Bases for its appropriate application in parafin-embedded tissues. Histochem Cell Biol (in press).

8. Wainscoat IS, Fey MF: Assessmenr of clonality in human tumors: A review. Cancer Res 50: 1355 1360, 1990. 
9. Gale RE, Wainscoat IS. Clonal analysis using $X$-linked DNA polymorphisms. Br J Haemato 85:2-8, 3993.

10. Allen RC, Zoghbi HY, Moscley AB, Rosenblatr HM, Belmont IW. Methylation of $\mathrm{Hpd}$ Il and Hhat sites near the polymorphic CAC; repeat in the human androgene receptor gene cortclates with $X$ chromosome inactivation. Am J Hum Gener 51:1229-1239, 1992.

11. Fialkow PJ. Primordial cell pool size and lineage relationships of five human cell types. Ann Hum Gener 37:39-48, 1973.

12. Kappler ]W. The 5-methyloytosine content of DNA: tissue specificity. ] Cell Physiol 78:33 36, 1971.

13. Latham KE. $X$ chromosome imprinting and inacrivation in the early mammalian embryo. TIG 12:134-137, 1996.

14. Page Bl, Delellis RA, Hough AJ Jr. Tumors of the adrenal. In: Arlas of tumor parbology, 2nd ser. fasci. 23. Washington DC: Armed Forces Instirute of larhology; 1986.

15. Lack EE. Tumors of the adrenal gland and extra-adrenal pacaganglia. In: Allas of tumor pathology, 3rd ser, Fasci. 19. Washingron $D C$ : Armed Forces Institure of Pathology: 1997.

16. Delellis RA, Wolfe HJ, Gagel RF, et al. Adrenal nedullary hyperplasia. A morphometric analysis in patients with familial anedullary thyroid carcinoma. Am I Parhol 83:177-190, 1976.

17. Carrey )A, Sizemore GW, Sheps SG. Adrenal medullary disease in multiple endocrine neoplasia, type 1 . Pheochromocytoma and its precursos. Am I Clin Pathol 66:279$290,1976$.

18. Neumann H3, Berger DP, Sigmund $G$, at al. Pheochomocytomas, multiphe endocrine neoplasia type 2, and von Hippel-Lindau disease. $N$ Engl ] Med 329:1531-1538, 1993.

19. Williamson EA, folunson SI. Foster S, Kendall-Taylor $\mathrm{P}$, Harris PE. G protein gene musacions in parients with muliplo endocrinopathies. I Clin Endocrinol Metab 80: $1702-1705,1995$.

20. Linnoila RI, Keiser HR, Steinberg SM, lack EF. Histopathology of benign versus malignant sympathoadenal paragangliomas. Clinicopathologic study of 120 cases including unusual histologic features. Hum Pathol $21: 1168-1180,3990$.

23. Diaz-Cano S\}, de Miguel M, Galera-Davidson $\mathrm{H}$, Wolfe $\mathrm{H}$. Are locally invasive pheochro- mocytomas biologically distinct from benign chromaftin ncoplasms? Pathol int 46 (Suppl 1): $223,1996$.

22. Diaz Cano SJ. Tashjian $R$, de Miguel M, et al. Distincrive clonal and histological pattens in locally invasive pheochromocyromas. I.ab Invest 76:153A, 1997.

23. Knudson $A G_{\text {. Antioncogenes and human }}$ cancer Proc Natl Acad Sci USA 90:10,914 $10,921,1993$.

24. Knudson AG. Mutation and cancer: A personal odyssey. Adv Cancer Res 67:1-23, 3995.

25. Gutmann DH, Cole JI., Stone W], Ponder $B A$, Collins FS. Loss of neurofibromin in adrenal gland tumors from patients with neurofibromatosis rype 1 . Genes Chromosom Cancer 10:55-58, 1994.

26. Khosla S, Patel VM, Hay ID, Schaid D], Grant $C S$, van Heeren IA, et as. loss of heterozygosity sngoests mulriple generic alterations in pheochromocytomas and medullary thyroid carcinomas. J Clin Invest 87:1691$1699,1991$.

27. Moley JF: Brothex MB, Fong CT, White PS, Baylin SB, Nelkin B, et al. Consistent association of 1 p loss of beterozygosity with pheochromocytomas from parients with multiple endocrine neoplasia type 2 syndromes. Cancer Res 52:770-774, 1992.

28. Prowse AH, Webster AR, Richard S, Olschwang $S$, Resche $F$, Affara NA, et al. Somatic inactivation of the VHL gene in von Higpel. Lindau disease rumors. Am I Hum Genes 60:765-771, 1997

29. Shin E, Fujita S, Takami K, Kurahashi H, Kurita Y, Kobayasbi T, et al. Deletion mapping of chromosome $1 p$ and $22 q$ in pheochromocytoma. Ipn I Cancer Res 84:402 408,1993

30. Vargas MP, Zhuang Z, Wang C, Vortmeyer A, Linehan WM, Merino MI. Loss of hetetozygosity of the shore arm of chromosome. 1 and 3 in sporadic pheochromocytomas and extra-adrenal paraganglioma. Hum Patbol 28:411-415, 1997.

31. Yokogoshi Y, Yoshimoto K, Saito S. Loss of heterozygosity on chromosomes $I$ and 11 in sporadic pheochromocyromas. Ipn ] Cancer Res 81:632-638, 1990 .

32. Boccia IM, Green JS, Joyce C, Fng C, Taylor SA, Mulligan LM. Mumition of RE I codon 768 is associated with the FMTC phenotype. Clin Gener 51:81-85, 1997 . 
33. Baylin SB, Hsu SH, Gam DS, Smalhidge RC, Wells SA Jr. Inherited medullary thyroid carcinoma: A fual monoclonal mutation in one of multiple clones of susceprible cells. Science $199: 429-431,1978$.

34. Bavlin SR, Gann DS, Hsu SH. Clonal origin of inherited medullary thyroid carcinoma and pheodromoycoma. Science 393 : $321 \cdots 323,1976$.

35. Woodruff MF Ansell JO Gorbes CM, Gondon C.. Burron DI, Micklem HS. Clonal interaction in tumours. Nature 299:822-824. 1982.

36. Knudson AC, Meadows AT. Regression of neuroblastoma IV'S: a genetic hyponbesis. N Fingl J Med 302:1254 -1256,1980.

37. Ferraris $A M$, Mangerini $R$, Gictani $G F$, Romei $C$, Pinchera $A$, Pacini I- Polvelonal origin of medullary catcisoma of the thyroid in mulriple endorrine neoplasia rype 2. Hum Genet 99:202-205, 1997.

38. Chen 1. , Kurisu W, Yjung BM, Goldman ES, Moor D 1l. Smith HS. Heterogeneity for allelic loss in human breast cancer. I Nat Cancer Inst 84:506-510, 1992.

39. Deng G, Le Y, Zlomilion G,Thor AD, Smith HS. loss of hercorygosity in nomal tissue adjacent to bralst carcinomas, Science $274: 2057-2059,3996$.

40. Wolman SR, Hepper GH. Genetic heterogencity in breast cincer. I Nat Cancer Inst $84: 469-470,1992$.

41. Sager R. Tumor suppressor genes: The puzhe and the promise. Science 246:140(1-1412.

42. Weinberg RA. Tumor suppressor genes. Science $254: 1138.1146$.

43. Smich HS. Stochastic model for interpreting the data on loss of heterozggosicy in breast cancer. I Nad Cancer lust 82:793-794, 1990 .

44. Mukahy GM, Goggins M, Wills $D$, et al. Pathology and genetic resting. Workshop No. 6. Cancer 80:636-648, 1997.

45. Decker RA, Peacock MI. Update on the probike of multiple endocrine aeoplasia type 2 a REX mutarions. Practical issues and implications fon genetic testing. Cancer 80:557-568, 1997 .

46. Versteeg R. Abertant methylation in cancer. An J Hum Gener 60:751-754, 1997.

47. Laird PW' Jienish R. DNA merbylation and cancer. Hum Mol Gener 3:3,487-1495, 1994.

48. Eng C. The RET proto-oncogene in multiple endocrine neoplasia type 2 and Hirschsprung's discase. N Engl ] Med $335: 943 \ldots 951,1996$.

49. Tischler AS, Delellis RA, Nunnemacher C, Wolfe [1]. Acute stimulation of chromafion cell proliferation in the adult rat adrenal medulla. Lab lnvest 58:733-735, 1988.

50. Tischler AS, Ruzicka LA, Donabue SR, Delellis RA. Tharmacological stimulation of chromaffin cell proliferation in the adult adrenal medulla. Arch Histol Cytol 52 (Suppl): $209-216,1989$.

51. Tischler AS, Ruricka LA, Van Pelt CS. Sandusky GE. Catecholamine-synthesining enzymes and chromogranin proteins in druginduced proliferavive lesions of the rat adrenal medulla. lab Invest $63: 44 \ldots 51,1990$.

52. Tischler AS, MCCkin RM, Childers H, Downing I. Neurogenic signals regukate chromaftin cell proliferation and mediate the mitogenio effect of reserpine in the adul rat adrenal medulla. Lab lnvest 65:374-376, 1991 . 